\title{
Advanced Sensor Technologies for Nondestructive Testing and Evaluation and Structural Health Monitoring
}

\author{
Gui Yun Tian,, ${ }^{1,}$ Lalita Udpa, ${ }^{3}$ Raimond Grimberg, ${ }^{4}$ B. P. C. Rao, ${ }^{5}$ and Shenfang Yuan ${ }^{6}$ \\ ${ }^{1}$ School of Electrical and Electronic Engineering, Newcastle University, Newcastle upon Tyne, NE1 7RU, UK \\ ${ }^{2}$ School of Automation Engineering, University of Electronic Science and Technology of China, Chengdu 611731, China \\ ${ }^{3}$ Department of Electrical and Computer Engineering, Michigan State University, East Lansing, MI 48824, USA \\ ${ }^{4}$ Nondestructive Testing Department, National Institute of Research and Development for Technical Physics, 47 D. Mangeron \\ Boulevard, 700050 Iasi, Romania \\ ${ }^{5}$ Metallurgy and Materials Group, EMSI Section, NDE Division, Indira Gandhi Centre for Atomic Research (IGCAR), Kalpakkam 603 \\ 102, India \\ ${ }^{6}$ The Aeronautic Key Lab for Smart Materials and Structures, Nanjing University of Aeronautics and Astronautics Nanjing 210016, \\ China
}

Correspondence should be addressed to Gui Yun Tian, g.y.tian@ncl.ac.uk

Received 25 June 2012; Accepted 25 June 2012

Copyright (c) 2012 Gui Yun Tian et al. This is an open access article distributed under the Creative Commons Attribution License, which permits unrestricted use, distribution, and reproduction in any medium, provided the original work is properly cited.

The need for monitoring the condition of critical structural components, that is, structural health monitoring (SHM), combined with increased requirements for the safety and quality of components, has resulted in the development of nondestructive testing and evaluation (NDT\&E) and nondestructive evaluation (NDE) methods for characterising materials and detecting the presence of defects. Defects and abnormalities such as corrosion, residual stresses, and microstructural changes may result in the structural integrity being compromised and an increased likelihood of failure. NDT\&E and SHM provide a means of identifying this damage and are often the only means of obtaining information about the current "health status" of a structure, through the installation of appropriate novel sensor technologies. It is our great pleasure to introduce the special issue in this emerging area to readers.

This special issue is devoted to the important area of sensor technology for NDT\&E and SHM, aiming to cover diverse aspects such as novel sensing methods, smart structures, sensor arrays and distributed sensors, advanced signal processing for sensor systems, and sensors for device, structural, and environmental monitoring. The papers in this issue demonstrate applications in monitoring of material properties, monitoring of advanced materials (composites), wireless sensor networks, and structural health monitoring.
The issue contains several papers dealing with the NDT\&E of various types of ferromagnetic components. The paper by I. J. Garshelis and G. Crevecoeur presents a novel method to measure hysteresis properties of ferromagnetic sheets using the magnetic field measurements measured by a magnet moved in the forward and reverse directions, where differences between these two measurements provide information on material properties affecting hysteresis. The paper by G. Psuj et al. presents a novel eddy current sensor for the detection of degradation in duplex stainless steel caused by an increase in sigma phase making the material more paramagnetic. W. S. Singh et al. present a paper on array of giant magnetoresistance (GMR) sensors to monitor the condition of steel track ropes. In this case the sensor array is constructed on a flexible PCB to wrap around the rope and detect flaws in and erosion of the steel rope. The paper by $\mathrm{H}$. Lei et al. presents a method to monitor the health of steel belts used in elevators by measuring the resistance of the steel ropes within the belts. This technique allows monitoring of the stress fatigue present in the rope from resistance changes.

Two papers present work on inspection of composite materials. The paper by L. Cheng and G. Y. Tian shows how both flash thermography and the recently developed PEC thermography techniques can be used to identify delamination within carbon fiber reinforced polymer components. An 
invited paper by T. Chady et al. presents a study of using pulsed terahertz $(\mathrm{THz})$ and thermography measurements to detect impact damage in glass-fiber reinforced composites.

This special issue contains two papers on work relating to wireless sensor networks. The paper by O. M. Bouzid et al. presents a novel signal processing method for wireless sensor networks. Envelope and wavelet transforms are used to enhance the resolution of data from the sensors when low sampling rates are used. The paper by P. Wang et al. presents an overview on the use of wireless sensor networks for SHM, specifically for monitoring offshore wind farms. The paper deals with issues related to monitoring of different types of components and the integration of sensor nodes into a network.

The paper by S. D. Boone et al. presents a novel sensor for the structural health monitoring of concrete structures, measuring variations in longitudinal compression wave velocity and energy absorption in concrete structures, which have been subjected to fatigue loading.

\title{
Acknowledgments
}

Introducing this special issue to the Journal of Sensors, we would like to thank all the authors for their contributions and reviewers for their help in achieving a high technical quality of papers in this special issue. The Lead Guest Editor Professor G. Y. Tian would like to thank all the Guest Editors' for their valuable contribution to this issue in particular. We hope that you enjoy the papers in the special issue as we do.

\author{
Gui Yun Tian \\ Lalita Udpa \\ Raimond Grimberg \\ B. P. C. Rao \\ Shenfang Yuan
}



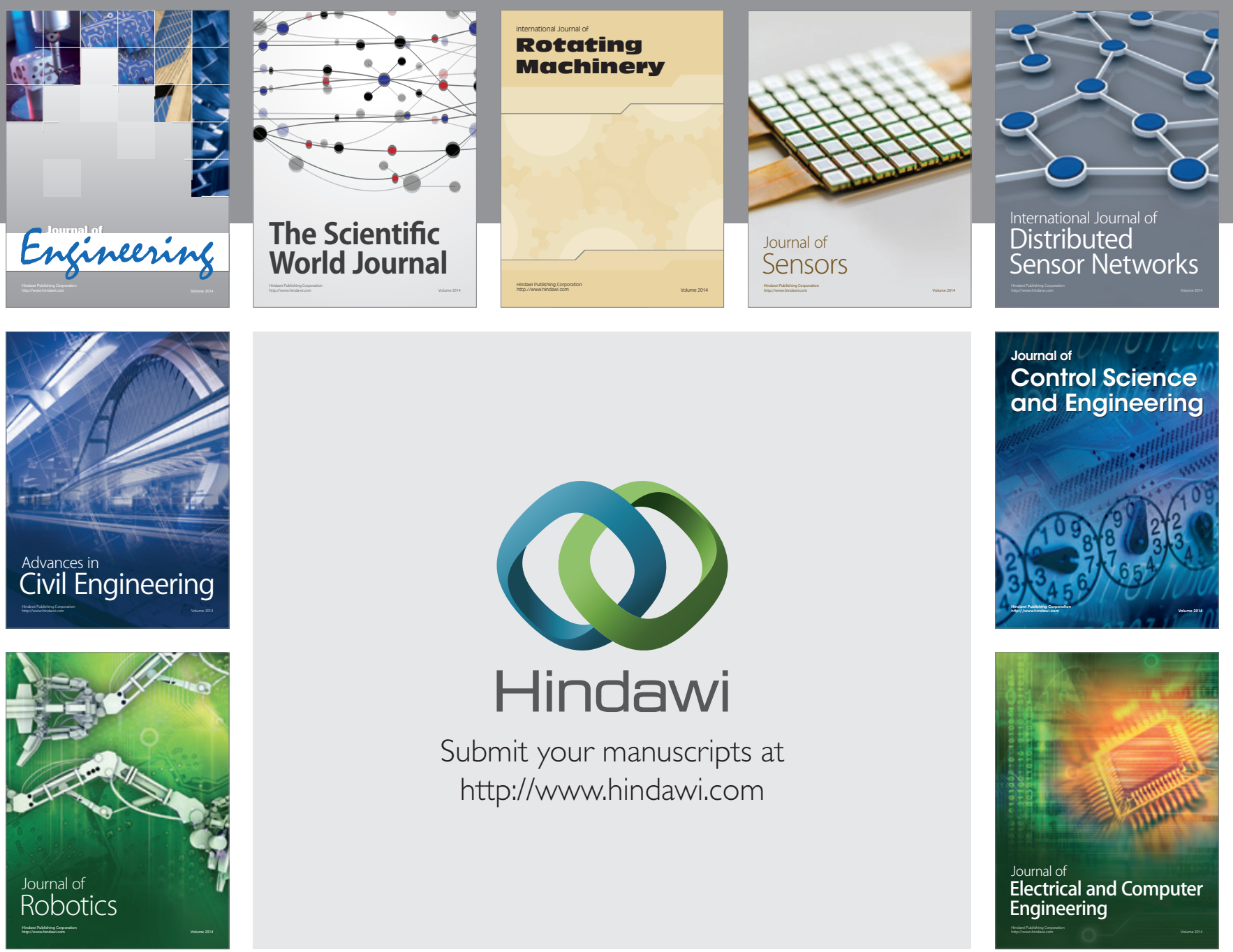

Submit your manuscripts at

http://www.hindawi.com
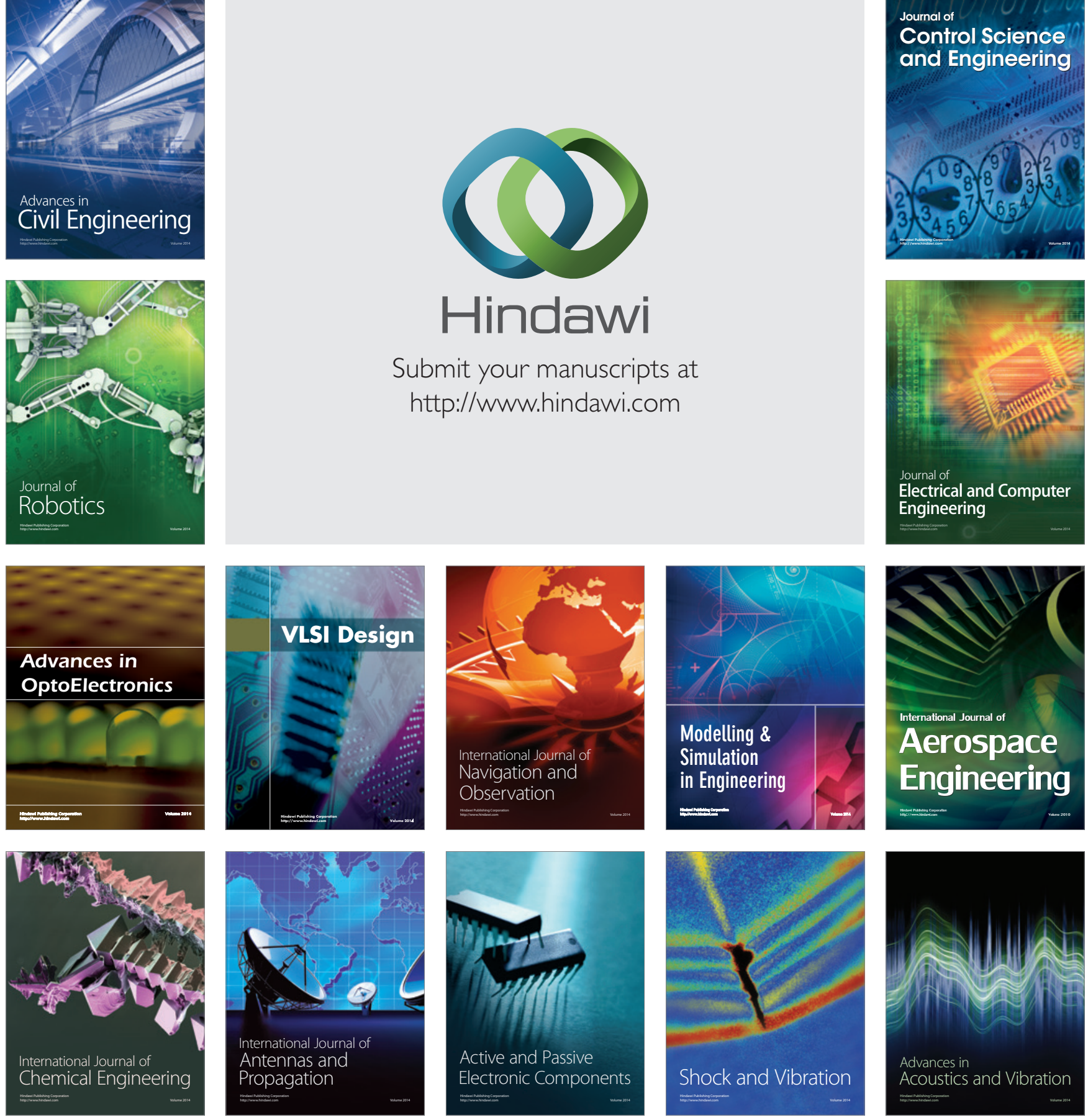